\title{
IDENTIFICATION OF THE OBSTACLE SHAPE USING THE Ultrasonic MOdUle OF MOdUlaR MOBILE ROBOT
}

\author{
Victor Andreev, Victoria Tarasova
}
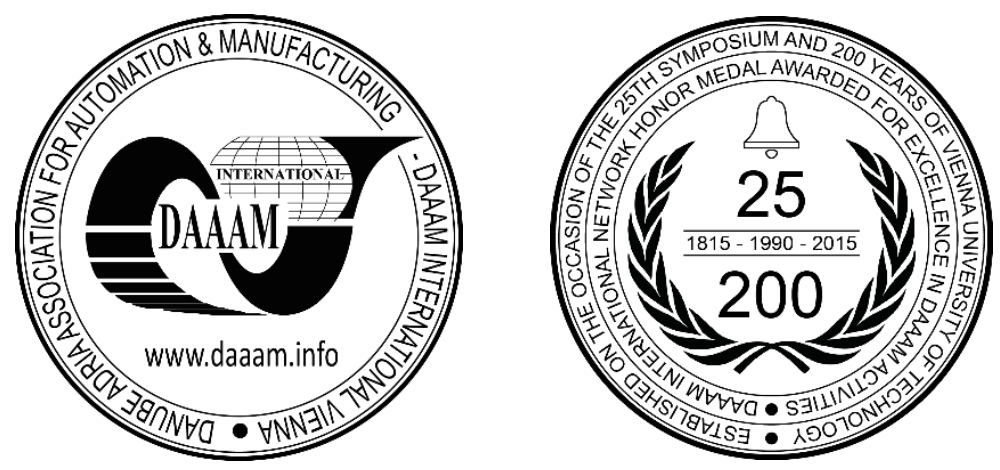

This Publication has to be referred as: Andreev, V[ictor] \& Tarasova, V[ictoria] (2018). Identification of the Obstacle Shape Using the Ultrasonic Sensors Module of Modular Mobile Robot, Proceedings of the 29th DAAAM International Symposium, pp.1001-1009, B. Katalinic (Ed.), Published by DAAAM International, ISBN 978-3-90273420-4, ISSN 1726-9679, Vienna, Austria

DOI: $10.2507 / 29$ th.daaam.proceedings. 143

\begin{abstract}
This paper shows the results of the study, the purpose of which is to solve the problem of identifying the shape of an obstacle and its orientation in a certain coordinate system using a sensor system of a modular mobile robot consisting of ultrasonic sensors. . Two implementations of the sensor system are considered: the ultrasonic sensor is located on the pivot-tilt mechanism controlled from a microcontroller; a multitude of ultrasound sensors is located along the perimeter of the robot sensor module. In the first case, the form of the obstacle is determined by scanning the surrounding space with one sensor; in the second case, all ultrasonic sensors are simultaneously firing and, based echo analysis the relief of the surrounding space is constructed, thereby performing a parallel scanning over the range of the territory. Experiments are show that the echo analysis of ultrasonic sensors with using the microcontroller is able to determine not only the azimuth of the obstacle, but also its shape such as the external (convex) corner, the internal (concave) corner, the flat wall, the doorway with the open door, the object of round shape and etc., and their orientation in some coordinate system.
\end{abstract}

Keywords: ultrasonic sensor; sensor system; mobile robot; modular robot; information measuring and control system.

\section{Introduction}

One of the problems of sensing a mobile robot (MR) is the creation of a sensory system to detect both static and dynamic obstacles, determine their location relative to the robot, and also their identification and orientation in some coordinate system. In the modular mobile robot, the sensor system is located to a separate part - the sensor module [1]. Depending on the surrounding in which the robot performs its tasks, it is necessary to be able to identify the various objects inherent in this environment and determine their location in relation to the robot. Therefore, in other words, it dynamically mapping of the surrounding. Thus, for orientation within a typical room, it is sufficient for the robot to identify the following three types of objects: planes (for example, walls), corners (both internal and external, formed by walls and supports) and doorways (with both open and closed doors). 
In [2], an example of the interaction of a transport and a sensor module is given for solving a typical problem of moving a modular robot from the initial position to the target one for the case of a lack of a surrounding map and if it exists. In both cases, the problem solved by the sensor module can be represented as follows. There is some room, within which the MP must move. The robot must achieve a pre-defined target to accomplish the task. The transport module moves to the target position, avoiding obstacles if necessary, for the detection and identification of which it uses the data of the sensor module. In that regard, it is necessary to determine the methodology for processing information about the environment. Then the following series of problems, conditions and limitations arise:

- The MP must dynamically determine its position in space for positioning relative to external objects and the target

- It is necessary to dynamically map the surroundings, identify static obstacles, avoid them and plan path towards the doorway

- Consider the possibility of dynamic obstacles

In most cases, to solve the problem of SLAM (simultaneous localization and matching) one uses the readings of sensors such as TV cameras or scanning devices that have high resolution. However, due to large data flows of coming from such devices, their use in mobile robotics is limited by the computing power of the on-board computer. Therefore, other sensors, such as ultrasonic range finders, replace these devices.

The problem is that the ultrasonic sensor has a wide beam aperture, i.e. large measuring angle $\left(\sim 30^{\circ}\right)$. Therefore, such a sensor can only detect an obstacle and determine the distance to some part of it. In addition, it is not known from what part of the obstacle an "echo" is received, and uncertainty arises in determining the distance to the obstacle. In the case of several objects overlapping the "visibility" zone of the sensor (Fig. 1), the distance can be determined, both up to the object one in front of the sensor (for example, point 2) and to the nearest point of object two (point 1). Consequently, because of the low resolving power of the sensor over the space, it is not possible to determine the shape of the obstacle that arises in the path of movement of the MR. At the same time, when moving robots in the space of residential or office space, it would be desirable to identify obstacles such as "corners formed by walls" (internal or external), or "doorways" (with doors open or closed).

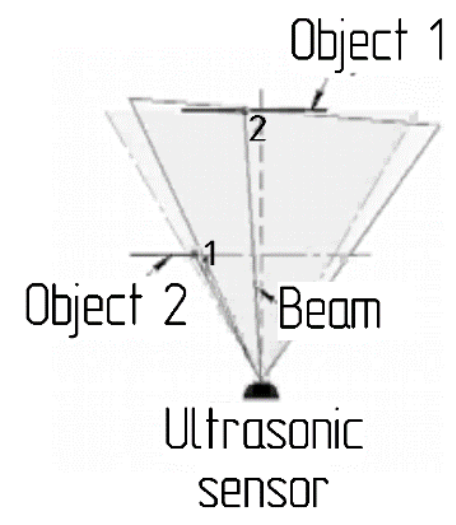

Fig. 1. Uncertainty in measuring distance sonar

As shown in [3], [4], [5], [6], [7], object type identification can be performed using a single scanning sensor. In these articles, experiments with a very limited set of forms are given. In addition, for the organization of scanning complex mechanism is used or the movement of the robot is used. This, in turn, affects the complexity of signal processing algorithms and leads to significant restrictions on the speed of the robot.

It is needed to find a solution that allows increasing the resolution of the ultrasonic sensor so that it can be used to not only detect obstacles and determine their type, but also to identify their shape. To solve this problem, it is proposed to use the micro scanning of the surrounding space with an ultrasonic sensor, by analogy with the drift movements of the human eye. Such a solution will significantly simplify the signal processing algorithms and, accordingly, will make it possible to use embedded systems - microcontrollers or single board computers - to solve the SLAM problem (under certain restrictions). As shown in [1], the use of embedded systems plays a significant role in creating mobile robots with a modular architecture.

\section{Use of scanning angular movements of the ultrasonic sensor}

To determine the shape of the obstacles, it is proposed to use the scanning angular displacements of the ultrasonic sensor [8]. When the sensor rotates, the field of view is shifted, resulting in point one (see Fig. 1) moving out of the beam aperture zone, and point two becomes the closest point. Calculating the difference between the measured distances, one can get a shape of the obstacle by approximating the values obtained with a function. To organize this movement, the ultrasonic sensor should be placed on a pivot-tilt mechanism, which is controlled from the microcontroller. 


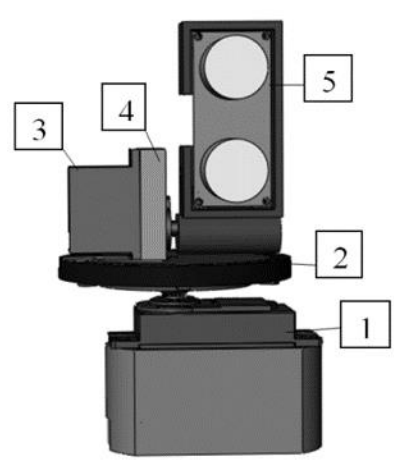

a)

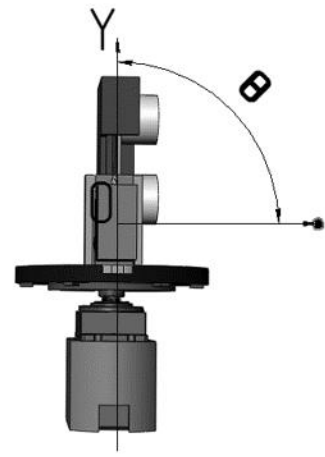

b)

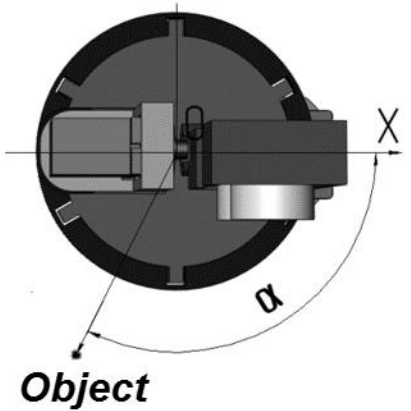

c)

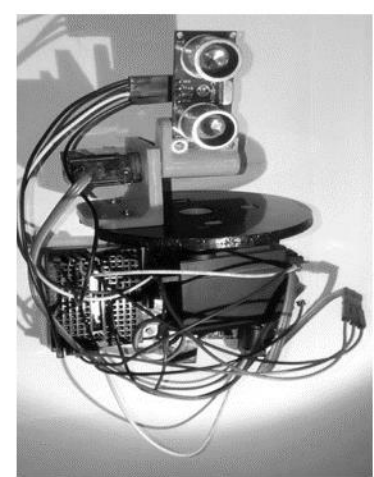

d)

Fig. 2. Device with ultrasonic sensor: a) front view; b) side view; c) top view; d) picture of the device

Fig. 2 shows a schematic representation of a pivot -tilt mechanism with an ultrasonic sensor. The mechanism consists of the following elements: 1 - servo FS5106B; 2 - turntable; 3 - servo SG90 9G; 4 - corner; 5 - UZ Sensor HCSR04. Also in the figure, $\alpha$ - the azimuth, the angle between the OX axis and the direction to the object; $\theta-$ the angle between the axis OY and the direction to the object.

The information output of the ultrasonic sensor is connected to the microcontroller Arduino Uno, which the duration of the pulse formed by the ultrasonic sensor is converted into a distance, measured in centimeters. Servo is controlled from the microcontroller too. The microcontroller is connected to the computer using of USB bus. The corresponding software allows you to control the servo, which accordingly change the orientation of the ultrasonic sensor in the space with an accuracy of $1^{\circ}$.

The computer sends a signal to the microcontroller, which determines the angle $\alpha$ of the rotation of the servo shaft (1), changing discretely with step $\Delta \varphi_{1}$ (horizontal scanning step). After each rotation, an ultrasonic sensor measured the distance to the object located in front of the sensor. As a result, a string is formed with the values of the measured distances corresponding to the rotation angle $\alpha$ from - $\alpha$ max to $\alpha$ max in the horizontal plane: $\Delta d=\left|d_{\mathrm{i}}-d_{\mathrm{i}+1}\right|>\mathrm{T}$, $\mathrm{T}$ - certain threshold indicating that the object ends. In this way, you can determine the width of the object in front of us and the angle of the object to the $O X$ axis of the device.

Where, according to our calculations, the middle of the detected object is located, we perform a vertical scan similar to the horizontal. The computer gives a signal cornering $\theta$ of the servo (2) shaft, changing discretely with step $\Delta \varphi_{2}$ (vertical scanning step). As a result, a column is formed with distances corresponding to the rotation angle $\theta$ from - $\theta_{\text {max }}$ to $\theta_{\max }$ in the vertical plane: $d=f(\theta)$. In this way, you can determine the height of the object in front of us and the angle of the object to the OY axis of the device.

\section{Experimental results}

The purpose of the experiments described below is to experimentally prove the possibility of using micro-movements of an ultrasonic sensor to determine the shape of relatively simple objects and their identification.

\subsection{Identification of a flat obstacle located vertically and oriented at some angle to the sensor (Fig.3)}

Scanning is from left to right. The object is at a distance $d=20 \mathrm{~cm}$, the scanning angle is $\alpha_{\max }= \pm 30^{\circ}$ with the step $\Delta \varphi_{1}=3^{\circ}$. The results are shown in Table 1 .
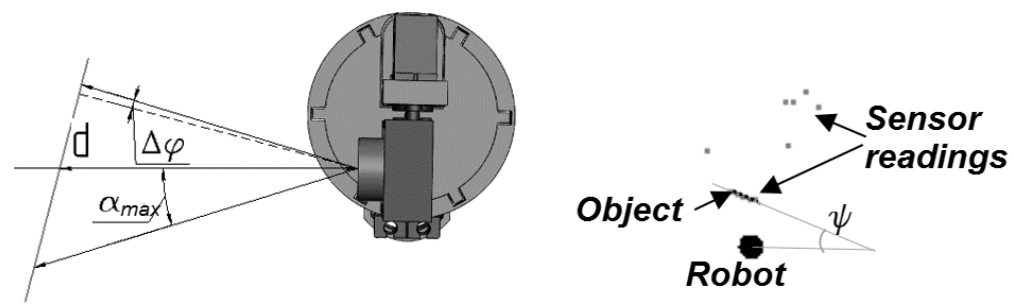

Fig. 3. Flat obstacle scanning scheme and distance measurement results (red marks)

\begin{tabular}{|c|c|c|c|c|c|c|c|c|c|c|c|c|c|c|c|c|c|c|c|c|c|}
\hline $\boldsymbol{\alpha}, \mathbf{d e g}$ & -30 & -27 & -24 & -21 & -18 & -15 & -12 & -9 & -6 & -3 & 0 & 3 & 6 & 9 & 12 & 15 & 18 & 21 & 24 & 27 & 30 \\
\hline $\boldsymbol{D}_{\boldsymbol{s}}, \mathbf{c m}$ & 54 & 28 & 26 & 25 & 24 & 25 & 24 & 25 & 23 & 21 & 21 & 18 & 18 & 16 & 16 & 15 & 56 & 78 & 77 & 85 & 70 \\
\hline
\end{tabular}

Table 1. Readings of the ultrasonic sensor $\boldsymbol{D}_{\boldsymbol{s}}$ at various angles of rotation 


\section{Algorithm:}

1) We exclude anomalous values of the measurements (points giving a sharp range difference with neighboring ones), as a result only the points making up the object

2) We approximate the points using the method of least squares (OLS), and determine the tilt angle (see Fig. 3)

The robot's action: the robot can turn to this angle and move parallel to the obstacle.

Conclusion. Under specified conditions, the identification of an object of the "flat obstacle" type is performed successfully.

\subsection{Identification of external (convex) angles}

Scanning is done from the left to the right (Fig.4). The object is at a distance $d=10 \mathrm{~cm}$, the scanning angle is $\alpha_{\max }= \pm 30^{\circ}$ with the step $\Delta \varphi_{1}=3^{\circ}$. The results are shown in Table 2 .

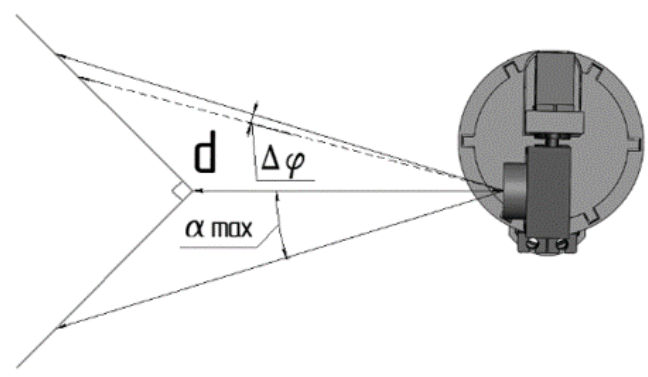

a)

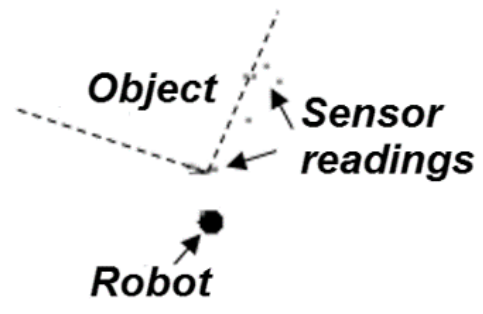

b)

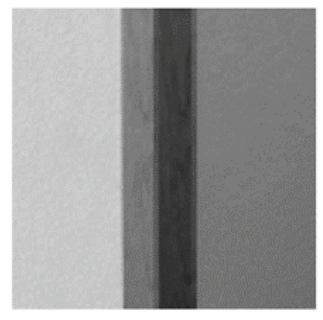

c)

Fig. 4. Scheme of scanning the outer corner (a), measurement results - red marks (b) and object's photo (c)

\begin{tabular}{|c|c|c|c|c|c|c|c|c|c|c|c|c|c|c|c|c|c|c|c|c|c|}
\hline $\boldsymbol{\alpha}, \mathbf{d e g}$ & -30 & -27 & -24 & -21 & -18 & -15 & -12 & -9 & -6 & -3 & 0 & 3 & 6 & 9 & 12 & 15 & 18 & 21 & 24 & 27 & 30 \\
\hline $\boldsymbol{D}_{\boldsymbol{s}}, \mathbf{c m}$ & 19 & 18 & 16 & 17 & 17 & 0 & 15 & 14 & 10 & 14 & 15 & 15 & 16 & 17 & 18 & 18 & 54 & 36 & 54 & 55 & 53 \\
\hline
\end{tabular}

Table 2. Readings of the ultrasonic sensor $\boldsymbol{D}_{\boldsymbol{s}}$ at various angles of rotation

Excluding points giving anomalous values, we get the following line (Table 3):

\begin{tabular}{|l|l|l|l|l|l|l|l|l|l|l|l|l|l|l|l|}
\hline $\boldsymbol{D}_{\boldsymbol{s}}, \mathbf{c m}$ & 19 & 18 & 16 & 17 & 17 & 15 & 14 & 10 & 14 & 15 & 15 & 16 & 17 & 18 & 18 \\
\hline
\end{tabular}

Table 3. Identifying the object

Scanning is performed from left to right:

1) First there is a decrease in the values $(19,18,16,17,17,0,15,14,10)$ - this is the first side of the angle

2) Then a gradual increase in the values $(15,16,17,18)$

Analysis result: decrease $=>$ increase in values, therefore, this is a convex angle.

Conclusion. Under specified conditions, the identification of an object of the type "external corner" is performed successfully.

\subsection{Identification of internal (concave) angles}

Scanning is done from left to right (Fig.5). The object is at a distance $d=10 \mathrm{~cm}$, the scanning angle is $\alpha_{\max }= \pm 30^{\circ}$ with the step $\Delta \varphi_{1}=3^{\circ}$. The results are shown in Table 4 .

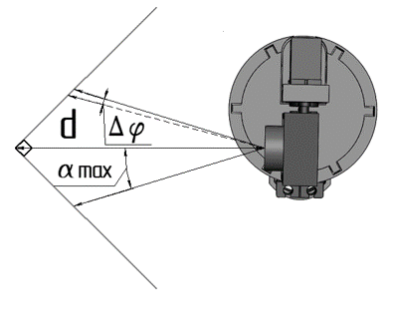

a)

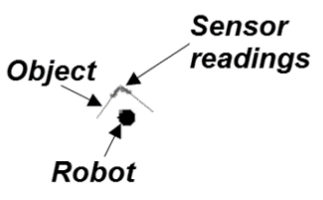

b)

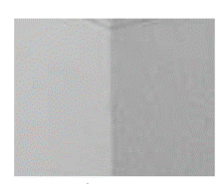

c)

Fig. 5. Scheme of scanning the internal corner (a), measurement results - red marks (b) and object photos (c). 


\begin{tabular}{|c|c|c|c|c|c|c|c|c|c|c|c|c|c|c|c|c|c|c|c|c|c|}
\hline $\boldsymbol{\alpha}, \mathbf{d e g}$ & -30 & -27 & -24 & -21 & -18 & -15 & -12 & -9 & -6 & -3 & 0 & 3 & 6 & 9 & 12 & 15 & 18 & 21 & 24 & 27 & 30 \\
\hline $\boldsymbol{D}_{\boldsymbol{s}}, \mathbf{c m}$ & 10 & 0 & 10 & 11 & 11 & 12 & 12 & 13 & 13 & 13 & 13 & 12 & 12 & 12 & 12 & 12 & 12 & 12 & 12 & 12 & 12 \\
\hline
\end{tabular}

Table 4. Readings of the ultrasonic sensor $\boldsymbol{D}_{\boldsymbol{s}}$ at various angles of rotation

Analyzing as described above, we conclude that this is a concave angle (increase-decrease values).

Conclusion. For a given conditions the identification of the "inner corner" object is performed successfully.

\subsection{Identification of "doorways"}

Scanning is performed when the door is fully open and when the door is not fully open (Fig. 6). Results of measurements (red marks): the object is at the distance $d_{1}=30 \mathrm{~cm}$ (Fig. 9 and Fig. 10); and $d_{2}=60 \mathrm{~cm}$ (Figure 11 ), the scanning angle $\alpha_{\max }= \pm 30^{\mathrm{O}}$ with the step $\Delta \varphi_{1}=1^{\mathrm{O}}$.

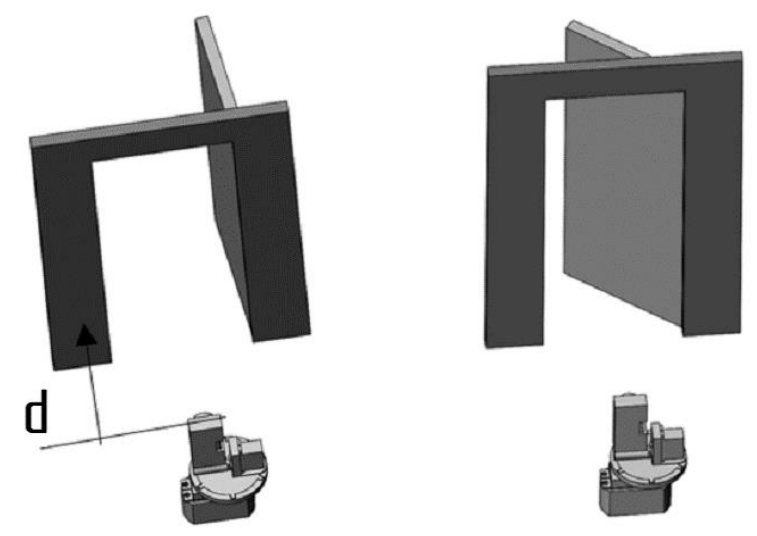

Fig. 6. The open door scanning scheme: fully open door (left); ajar door (on the right)

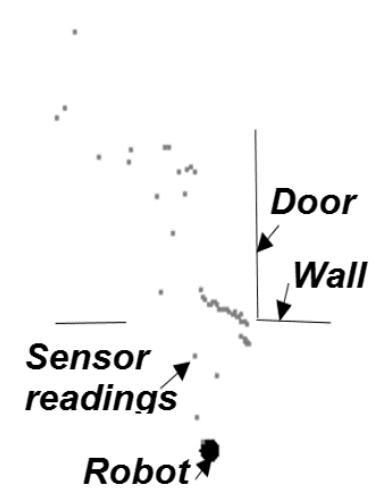

a)

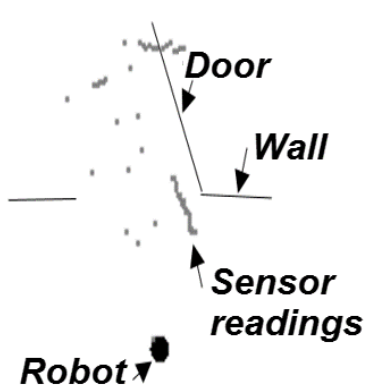

b)

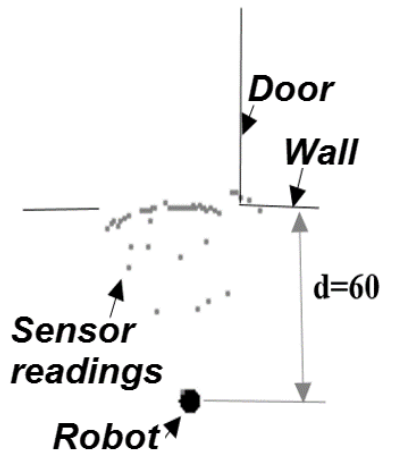

c)

Fig. 7. Open door, $d_{1}=30 \mathrm{~cm}(\mathrm{a})$; the slightly open door $\mathrm{d}_{1}=30 \mathrm{~cm}(\mathrm{~b})$; distance $\mathrm{d}_{2}=60 \mathrm{~cm}(\mathrm{c})$.

The robot in the distance more than $50 \mathrm{~cm}$ from the doorway (width $\mathrm{d}_{2}=60 \mathrm{~cm}$ ), does not detect the doorway (Fig. $7 \mathrm{c}$ ). Conclusion. For a given conditions the identification of objects such as «open door» and «the slightly open door» is successful.

\section{Ultrasonic sensor system of parallel firing}

Scanning of the surrounding area by one sensor requires obtaining a multitude of measurements in different directions, and the resolution of such a device depends on the number of firing-echo cycles and the angular velocity of the scanning device. Each such cycle takes time depending from the sensor design. This, in turn, significantly affects the speed of the robot. This disadvantage can be avoided if several sensors are simultaneously used - oriented in different directions. It is possible for one sensor's ultrasonic wave to affect readings from the other sensor because they all operate at one resonant frequency, so that it may not be possible to identify the obstacle shape. Articles [9], [10], [11], [12] show experiments with the simultaneous or sequential firing of several ultrasound sensors to identify obstacles, but in these works complex systems of digital processors are used to trigger sensors and echo analysis. 
The purpose of the experiments described below is to experimentally prove the possibility of using an ultrasonic sensor system of parallel action, consisting of a set of simultaneously operating ultrasonic distance sensors, to determine the shape of simple objects and their identification.

The following objects were used as objects of the simplest forms: a round (surrounding) wall, a flat wall and an external (convex) angle formed by the walls. A sensor module (Fig.8) with twelve ultrasonic sensors (it is a short-range sensor system of a mobile robot with a modular architecture) was developed and manufactured [13]. It is assumed that the simultaneous operation of ultrasonic sensors located around the circumference of the sensor module with equal steps relative to each other $\left(30^{\circ}\right)$ will not affect the results of the identification of the shapes of objects.

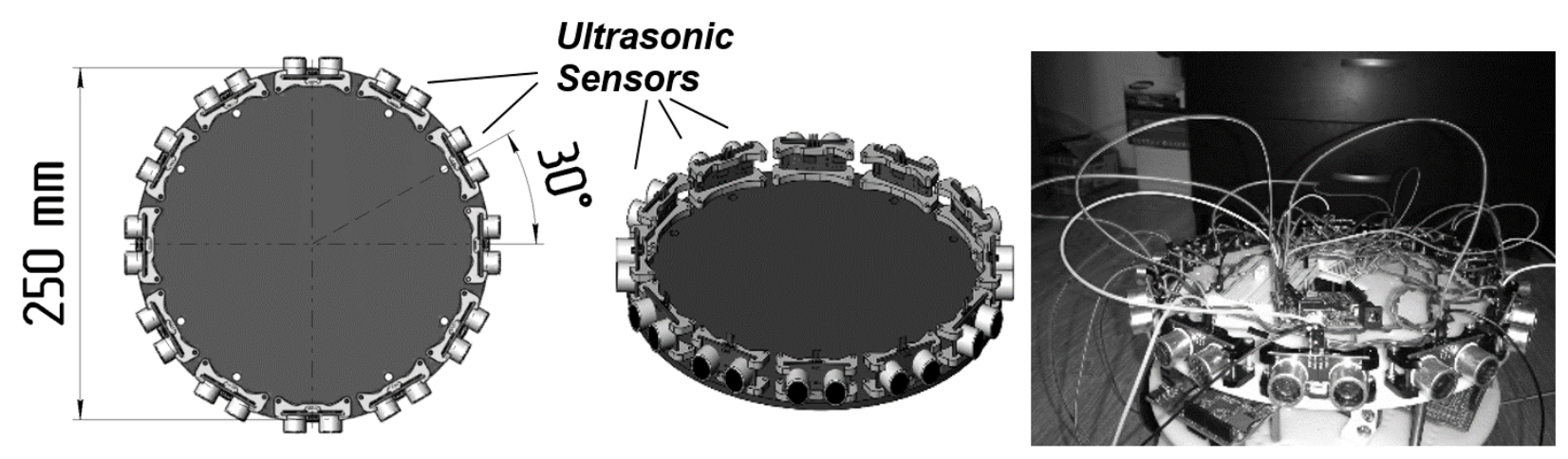

Fig. 8. The design of an ultrasonic sensor module and a photo of a modular mobile robot with a parallel-action sensor

\subsection{Identification of a round shape obstacle (Fig. 9)}

The results are shown in Table 5.

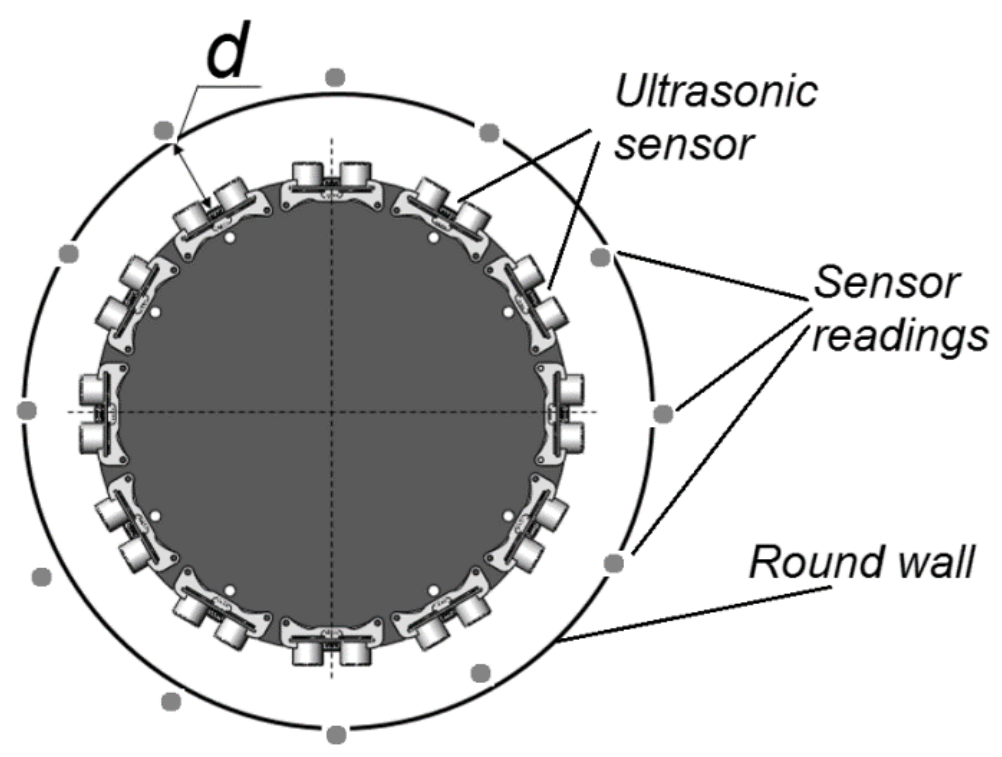

Fig. 9. Scheme of identification of a round shape $(d=20 \mathrm{~cm})$

\begin{tabular}{|c|c|c|c|c|c|c|c|c|c|c|c|c|}
\hline Sensors & $\mathbf{1}$ & $\mathbf{2}$ & $\mathbf{3}$ & $\mathbf{4}$ & $\mathbf{5}$ & $\mathbf{6}$ & $\mathbf{7}$ & $\mathbf{8}$ & $\mathbf{9}$ & $\mathbf{1 0}$ & $\mathbf{1 1}$ & $\mathbf{1 2}$ \\
\hline Mean value, $\mathbf{c m}$ & 20,2 & 19,7 & 16,4 & 23,3 & 18,5 & 19,2 & 25,4 & 22,3 & 17,9 & 18,1 & 19,2 & 20,1 \\
\hline
\end{tabular}

Table 5. Readings of the ultrasonic sensors

Conclusion. For a given conditions the identification of an object of the "surrounding obstacle" type is performed successfully.

4.2 Identification of a flat object located vertically and oriented at some angle to the direction of movement of the robot (Fig. 10)

The results are shown in Table 6. 


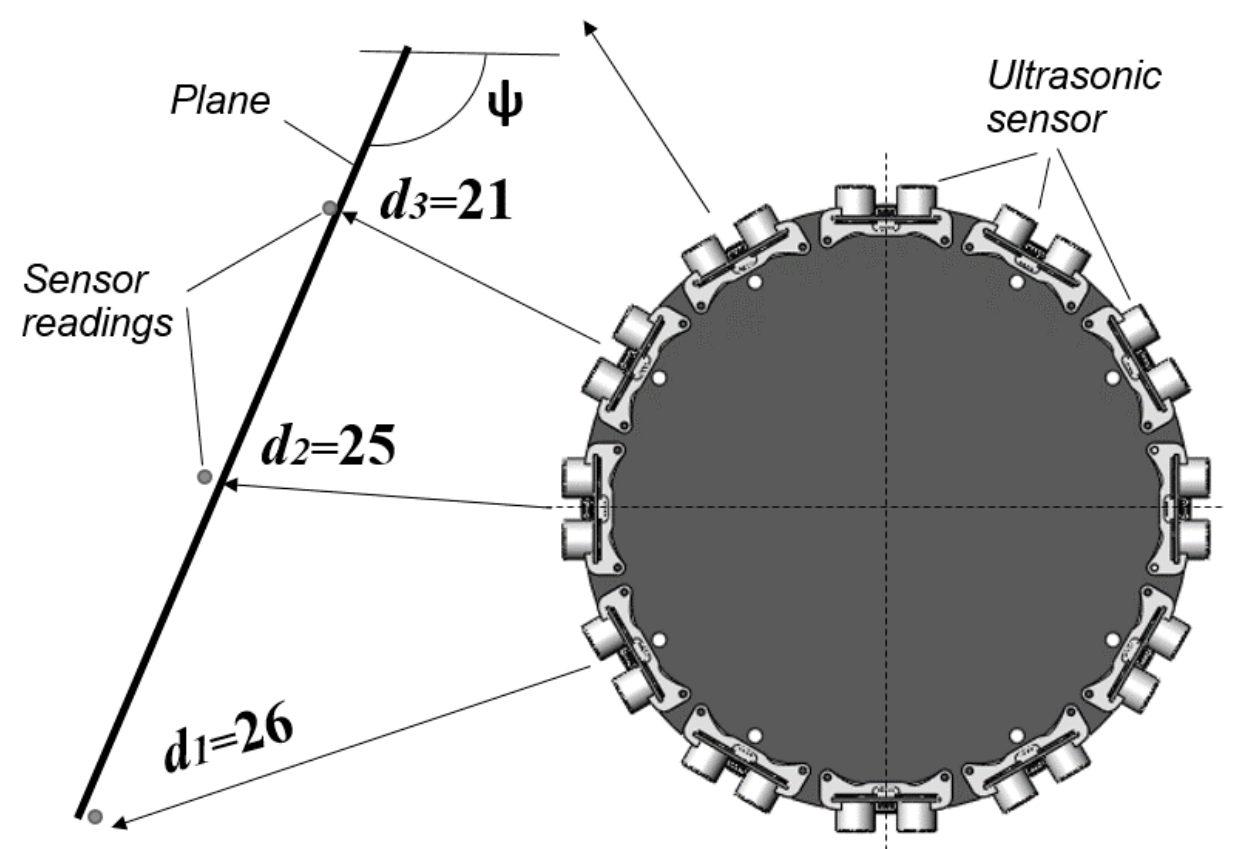

Fig. 10. Scheme of identification of a flat object located vertically and oriented at some angle to the direction of movement of the robot $(d-$ real distance values)

\begin{tabular}{|c|c|c|c|c|c|}
\hline Sensors & $\mathbf{1}$ & $\mathbf{2}$ & $\mathbf{3}$ & $\mathbf{4}$ & $\mathbf{5}$ \\
\hline Mean value, $\mathbf{c m}$ & 85,4 & 25,6 & 23,5 & 21,3 & 68,5 \\
\hline
\end{tabular}

Table 6. Readings of five ultrasonic sensors

From the table the sensors 2, 3 and 4 "see" the obstacle, and a significant difference in the readings of the 1st and 2nd sensors, as well as the 4th and 5th allow us to say the boundaries of the object.

Conclusion. For a given conditions the identification of an object of the "flat wall" type is performed successfully.

\subsection{Identification of the external (convex) corner (Fig.11)}

The results are shown in Table 7.

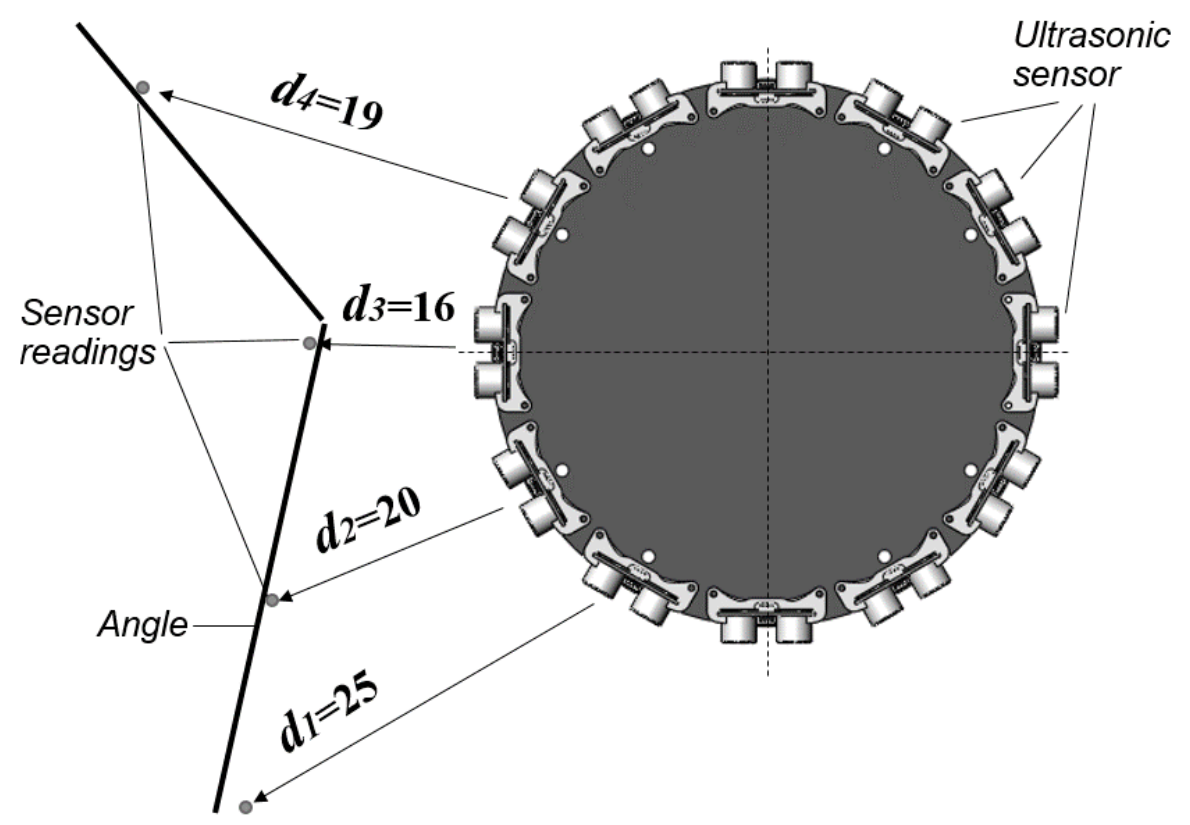

Fig. 11. Scheme of identification of the external corner 


\begin{tabular}{|c|c|c|c|c|c|c|}
\hline Sensors & $\mathbf{1}$ & $\mathbf{2}$ & $\mathbf{3}$ & $\mathbf{4}$ & $\mathbf{5}$ & $\mathbf{6}$ \\
\hline Mean value, $\mathbf{c m}$ & 54,5 & 21,2 & 18,7 & 16,2 & 18,3 & 67 \\
\hline
\end{tabular}

Table 7. Readings of six ultrasonic sensors

First, the readings of the 2, 3 and 4 sensors are reduced - this is the first side of the angle; then there is a consistent increase in the readings of the fifth sensors - this is the second side of the angle. A significant change in the indications of the first and the second sensors and the fifth and sixth, allow us to say the boundaries of the object.

Analysis result: decrease $=>$ increase in values, hence, this is a convex corner.

Conclusion. For a given conditions the identification of an object of the type "external corner" is performed successfully.

\section{Conclusion}

Experiments have shown that the use of scanning angular displacements of the ultrasonic sensor allows determining the shape of an obstacle such as "external corner", "internal corner", "flat wall", "doorway with the door open", "the slightly open door" and their orientation some coordinate system. Analysis of various ways to identify the form of obstacles using an ultrasonic sensor system showed that mechanical scanning of space, performed sequentially, is effective, but it takes considerable time to analyze the environment, which makes it necessary to reduce the speed of movement of the mobile robot.

Simultaneous use of several sensors significantly speeds up the process of identifying the form of obstacles and reduces the scanning time of the medium, which makes it possible to significantly increase the speed of the robot, but the resolution decreases in comparison with the micro scanning method. The ring of ultrasonic sensors firing simultaneously allows parallel calculations and quick analysis of the surrounding space, and for a given conditions, the crosstalk of the ultrasonic sensors is not observed. However, with a certain form of obstacles, such an effect may occur, which will require the development of an algorithm for processing readings that filter «echo» from neighboring ultrasonic sensors.

In the future, it is planned to identify more complex shapes and sizes of obstacles, as well as work with dynamic objects. It is necessary to perform an approximation of the sensor readings and, as a result, to obtain an estimate of the "curvature" of the obstacle, the "convexity" or "concavity" of the corners. The range of micro scanning and its dependence on external factors should be determined.

\section{Acknowledgments}

The authors are expressing their sincere gratitude to S. Kuvshinov, Director of International Institute of the New Educational Technologies of Russian State University for the Humanities, for assistance in construction of the experimental equipment. The Russian Foundation supported this work for Basic Research, grants no. 16-07-00811a \& $1607-01264 a$.

\section{References}

[1] Andreev, V.; Kim, V. \& Pletenev, P. (2017). The principle of full functionality - the basis for rapid reconfiguration in heterogeneous modular mobile robots // Proceedings of the 28th DAAAM International Symposium, pp. 0023-0028, B. Katalinic (Ed.), Published by DAAAM International, ISBN 978-3-902734-11-2, ISSN 1726-9679, Vienna, Austria. DOI:10.2507/28th.daaam.proceedings.003.

[2] Andreev, V. \& Kim, V. (2017). The application of artificial vector fields for motion control of a heterogeneous modular mobile robot, Proceedings of the 28th DAAAM International Symposium, pp. 0635-0644, B. Katalinic (Ed.), Published by DAAAM International, ISBN 978-3-902734-11-2, ISSN 1726-9679, Vienna, Austria. DOI: 10.2507/28th.daaam.proceedings.090.

[3] Daru, R.; Biswas, H. \& Barman, S. (2016). Determining 2D Shape of Object Using Ultrasonic Sensor, Publisher, ISBN 978-1-5090-2906-8, Bangladesh.

[4] Crowley, J. (1985). Dynamic World Modeling for an Intelligent Mobile Robot Using a Rotating Ultra-Sonic Ranging Device, Proceedings of IEEE International Conference on Robotics and Automation, ISSN CH2152-7, pp. 128-135, Publisher, USA.

[5] Jayprakash M.,; Riyazuddin N.; Mudassir S. \& Niraj D. (2016). Development of Radar Using Ultrasonic Sensor, International Journal of Technical Research and Applications, pp. 99-101, Publisher, ISBN 2320-8163, India.

[6] Rodin R. \& Stajduhar I. (2017). The Challenge of Measuring Distance to Obstacles for The Purpose of Generation a 2-D Indoor Map Using an Autonomous Robot Equipped with an Ultrasonic Sensor, ISSN 978-953-233-090-8, pp. 1021-1026, Publisher, Croatia.

[7] Tashev A. (2017). The development of a robot based on an ultrasonic rangefinder, Available from: http://oreluniver.ru/file/chair/aplast/nol/TashevFedorov.pdf., Accessed: 2017-10-22. 
[8] Andreev V. \& Tarasova V. (2017). Determination of the Form of Obstacles by a Mobile Robot Using Scanning Angular Movements of Ultrasonic Sensor. Mechatronics, Automation, Control, Vol. 18, No. 11, pp. 759 -763, DOI: $10.17587 / \mathrm{mau} .18 .759-763$.

[9] Kleeman L. \& Kuc R. (1995). Mobile Robot Sonar for Target Localization and Classification. International Journal of Robotics Research, Vol. 14, pp. 295-318, USA.

[10] Klahold J.; Rautenberg J. \& Ruckert U. (2002). Continuous Sonar Sensing for Mobile Mini-Robots. International Conference on Robotics and Automation, ISSN 0-7803-7272-7, pp. 323-328, Germany.

[11] Vlastimil M.; Ming A. \& Vlacic L. (1998). A Fast Mobile Robot Obstacle Detection Using Simultaneous Firing of Sonar Ring Sensors. The Japan Society for Precision Engineering, Vol. 32, pp. 207-212, Japan.

[12] Fazli S. \& Kleeman L. (2004). A Real Time Advanced Sonar Ring with Simultaneous Firing, IEEE/RSJ International Conference on Intelligent Robots and Systems, ISSN 0-7803-8483, pp. 1872-1877, Japan.

[13] Andreev V.; Kim V. \& Pletenev P. (2018). Hardware \& Software Solution for Rapid Reconfiguration of Heterogeneous Robots, Mechatronics, Automation, Control, Vol. 19, No. 6, pp. C.387 - 395 , DOI: $10.17587 / \mathrm{mau} .19 .387-395$. 